\title{
Littérature politique et exégèe biblique (de 1570 à 1625)
}

PIERRE-LOUIS VAILLANCOURT

Le recours á la Bible

A la fin de la Renaissance, la Bible n'est plus au coeur de la pensée politique, mais elle reste l'instrument de sa justification. Les traités politiques se réclament sans cesse de la Bible pour confirmer la valeur de leurs théories. Même si la Bible est présentée comme la source apparente de celles-ci, elle sert plutôt à garantir leur validité. Les premiers écrivains de la Réforme, Luther, Zwingli, Calvin et Melanchthon avaient contribué, dans la première partie du seizième siècle, à redonner à la Bible une autorité éminente dans l'élaboration des concepts même sociaux et à assurer sa prépondérance sur les instances usuelles de la vérité: la tradition, la papauté, l'Eglise, la scolastique. Parole directe de Dieu, la Bible redevient la pierre de touche de tout savoir, le lieu de résolution de toutes les controverses. Si Luther a accordé la priorité au Nouveau Testament, Calvin et Zwingli mettront sur le même plan Ancien et Nouveau Testament, comme "règle unique de vraye et parfaite sagesse" (unica perfectae sapientiae regula). ${ }^{1}$ Très tôt, les dangers de cette orientation apparurent, et les paysans révoltés, rappelant à Luther qu'ils ne trouvaient pas dans la Bible la dîme du bétail à payer, obligèrent celui-ci à accorder l'inspiration divine et le contexte social. L'entreprise de la Réforme détermina cependant un respect prononcé pour la Bible qui s'étendit aux scolastiques catholiques, entrainés à répondre à la lettre des arguments de leurs adversaires par d'autres puisés au même fonds, et aux penseurs les plus laïcs, soucieux de réconcilier l'influence antique, les faits contemporains et l'enseignement biblique. Le rétablissement marqué avec éclat de la Tradition et de la Vulgate au Concile de Trente, témoigne a contrario de l'audience acquise par le livre sacré, sous l'impulsion des Réformés habitués à l'utiliser pour la recherche de toute vérité, religieuse ou politique.

Cette primauté reconnue astreint à de subtiles adaptations les auteurs de toutes tendances, mais en particulier ceux dont les principes temporels semblent l'emporter sur les spirituels. Il n'est pas aisé, ni possible, de juger erroné ou sans fondement une leçon biblique, comme pourrait l'être un 
jugement d'Aristote. La Bible impose une direction d'interprétation. Discutant de la punition des attentats commis contre François Ier et Henri II, Bodin termine sa démonstration par ces mots: "Et à fin qu'on ne die point que les hommes ont faict ces loix, \& donné ces arrests, nous lisons en la saincte Escriture, que Nabuchodonosor . . . " 2 Le texte biblique toujours appelé à corroborer une opinion ne peut jamais être explicitement contredit. Aussi Bodin dresse-t-il une liste des tyrans de la Bible envers lesquels les prophètes ont recommandé la soumission et ajoute: “'Il n'ya rien de plus frequent en toute l'escripture saincte, que la defense, non pas seulement de tuer y attenter à la vie ou à l'honneur du Prince: ains aussi des Magistrats, ores (dit l'Escriture) qu'ils soyent meschans." 3

Les partisans de la Réforme témoignent évidemment d'un attachement plus vif encore. Lorsque Bèze s'interroge à son tour sur les devoirs des sujets envers un roi devenu tyran, il passe en revue les prérogatives des citoyens de Rome, d'Athènes, du Danemark, d'Ecosse, de Lacédémone, d'Angleterre, de Pologne, de Venise, d'Espagne, en citant le rôle de différents corps pour limiter l'arbitraire du pouvoir, mais l'exemple également évoqué d'Israël commande un traitement particulier et plus élaboré. ${ }^{4}$ Il ne présente pas uniquement des exemples mais discute longuement des textes de la Bible consacrés aux monarchies divine et temporelle. Tous les théoriciens réformés partagent cette considération. Ainsi Buchanan multiplie les nuances sur les concepts de l'autorité séculière dans le Nouveau Testament. ${ }^{5}$ Languet, cherchant s'il est légal de résister à un prince qui viole la loi divine, énonce les principes qui le guident: "si nous nous en tenons au dire de l'Escriture saincte, elle nous en resoudra." "Lorsque plus tard Jacques Ier d'Angleterre désire tout au contraire prôner l'obéissance inconditionnelle et non pas la résistance conditionnelle, c'est au moyen de divers extraits de la Bible qu'il défendra le pouvoir des rois. ${ }^{7}$ Autre écrivain inspiré par la Réforme, Althusius utilise plus souvent la Bible que toute autre source parce qu'il croyait en la supériorité de l'organisation étatique juive. $^{8}$

Bien que plus liés à l'enseignement de l'Eglise et des Pères, les théoriciens scolastiques subissent l'influence de cette vénération. Juan de Marquez, religieux de l'ordre de saint Augustin et prédicateur de Sa Majesté catholique, donne ce titre éloquent à son traité: L'Homme d'Estat Chrestien, tiré des vies de Moyse et Josué Princes du peuple de Dieu. ${ }^{9}$

Et Suarez, une des gloires de la Compagnie de Jésus, apologiste des Pères et de la pédagogie ecclésiastique, apporte volontiers les diverses interprétations d'un passage biblique, pour les commenter, les réfuter ou en proposer de nouvelles. ${ }^{10}$ Absolutistes, monarchomaques huguenots et scolastiques exigent tous de la Bible une sanction favorable à leur parti pris.

Il est possible que tous les auteurs de cette époque n'aient pas eu une 
conscience vive des rôles multiples et parfois contradictoires que jouaient les références bibliques mais cette situation n'a pas échappé à la clairvoyance d'un Du Perron, par exemple, qui souligne, mais sans ironie, la difficulté d'asseoir une position ferme et nette sur la Bible, car elle est brandie par tous les partis.

Et donc quel article de foy ne sera point arraché du tribunal de l'Eglise, \& exposé en proye à la présomption des heretiques, s'il suffit de dire qu'il est si clair dans l'Escriture qu'il n'y eschet ny dispute ny jugement? A la vérité cela auroit quelque apparence, si ceux qui tiennent l'une des propositions alleguoient l'Escriture pour eux, \& que les autres ne l'alleguassent point. Mais tant ceux que tiennent l'affirmative, que ceux qui tiennent la negative, argumentent par l'Escriture, répondent par l'Escriture, \& repliquent par l'Escriture. ${ }^{11}$

Mais du Perron tombe à son tour dans cette habitude lorsque, discutant de la forme des gouvernements dans les premières cités humaines, il écrit: "Mais vray dire nul auteur gentil en peut avoir parlé avec certitude laquelle nous tirerons des sainctes escriptures."12

\section{La Bible devant l'état}

Nombreux sont les textes qui dans les deux Testaments entretiennent un rapport plus ou moins étroit avec la constitution des sociétés, mais aucun n'avance une théorie spécifique sur l'origine des communautés. La Chute et sa conséquence, la nature déficiente de l'homme, ont servi à justifier la nécessité d'un ordre politique mais ont aussi alimenté la méfiance augustinienne à l'égard d'un ordre établi par une faute et dans la violence: la cité d'Enoch ayant été fondée par Caïn, un fratricide. ${ }^{13}$ Cette carence théorique permet à saint Thomas d'intégrer les idées aristotéliciennes d'une tendance innée au lien social chez les hommes et d'une finalité bénéfique du pouvoir, supposant, pour sa formation et son maintien, l'intervention de Dieu comme causa remota. ${ }^{14} \mathrm{La}$ Bible ne présente pas non plus la théorie, chère à Bodin, d'une croissance naturelle de l'état à partir de la cellule familiale. Elle ne précise pas si les premiers rois de l'humanité ont été choisis ou se sont imposés par la violence. La Genèse mentionne brièvement que le premier potentat a été Nemrod, vaillant chasseur qui fonda un empire composé des villes de Babel, Ereq et Akkad. ${ }^{15}$ Seul est décrit en détail dans le Livre de Samuel l'établissement de la monarchie en Israël.

S'ils ne présentent pas, sauf dans Samuel une conception élaborée de la société et du pouvoir, les textes sacrés comportent cependant maints faits à valeur exemplaire, notamment les tribulations des Israélites, dans le désert et en exil, et de nombreux conseils, en particulier sur le comportement du chrétien à l'égard des autorités civiles dans les textes des apôtres Pierre et Paul. Par leur caractère limité et souvent conjoncturel, ces éléments se 
prêtent plus aisément qu'une théorie organique à des manipulations idéologiques.

\section{Choix des textes et conditions historiques}

Les emprunts faits à la Bible par la littérature politique, de Bodin à Grotius, sont déterminés par les bouleversements en cours. La croissance des monarchies séculières, au détriment des autorités impériale et pontificale, la fin des particularismes locaux, l'émergence des états nationaux modifient l'horizon politique général. D'une façon plus particulière, la StBarthélemy et l'avènement d'Henri IV en France, les dominations successives de souverains catholiques puis protestants en Angleterre et en Ecosse, avaient amené au centre des préoccupations l'obéissance ou la résistance des sujets en matière de conscience et de pratique religieuses. En ébranlant l'état, les guerres de religion avaient aussi fait naître en France un parti, celui des Politiques, voué aux intérêts de la monarchie temporelle contre les empiètements de la religion. Contesté ou renforcé, le pouvoir temporel devait se définir à l'égard du pouvoir papal. Ce fut le problème d'Henri IV, mais bien plus celui de Jacques Ier, lors du serment d'allégeance réclamé à ses sujets après la Conspiration des Poudres. D'éminents scolastiques comme Bellarmin et Suarez prennent la plume, à l'incitation de Rome, contre ce roi polémiste et ses partisans. Les variations, d'un règne à l' autre, entraînent l'échange des théories. Le climat de camps retranchés dans lequel vit l'Europe de l'Ouest et du Nord est fort propice à l'effervescence théorique, comme l'indiquent la prolifération des pamphlets, l'abondance (et la longueur) des traités politiques. Chaque camp pouvait trouver dans la Bible matière à contentement ou à préoccupation.

Les premières oeuvres marquantes de cette période furent écrites par les monarchomaques, tels Buchanan, Hotman, Bèze, Mornay, Languet et par les auteurs anonymes des textes publiés dans les Mémoires de l'Estat de France ${ }^{16}$ Les monarchomaques, ouvrant une brèche à la désobéissance civile, se heurtaient, dans la Bible, aux appels à la soumission des apôtres Pierre et Paul. Dans un texte clair et précis, saint Paul demande que chacun se soumette aux autorités en charge, en soulignant que toute autorité vient de Dieu. ${ }^{17}$ Toute rébellion est un rejet de Dieu. L'autorité étant instituée comme un instrument de justice, il convient de se soumettre tant par motif de conscience que par crainte du châtiment. Ce message se trouve renforcé par deux textes, l'un apparaissant dans une épitre de saint Pierre, ${ }^{18}$ l'autre dans l'Ancien Testament, lorsque Jérémie demande à son peuple d'obéir à Nabuchodonosor, malgré les torts de ce dernier envers les Juifs qu'il déporta et réprima. ${ }^{19}$ Néanmoins il est appelé le serviteur de Dieu et Jérémie presse son peuple d'ignorer les injonctions faites par les faux prophètes de ne pas se considérer assujettis au roi de Babylone. 
Bien sûr, l'épître aux Romains est l'un des textes les plus commentés, non seulement par les monarchomaques, mais par des absolutistes comme Jacques Ier et P. de Belloy, qui trouvent là un langage inespéré pour leur cause. La Bible est cependant tissée de contradictions; chaque allégation peut être confrontée à sa contradiction ou atténuée par diverses réserves. Un fragment d'une épître aux Corinthiens servira par exemple d'échappatoire.

L'Ancien Testament procure son contingent de textes. Des épisodes du livre de Daniel s'avèrent particulièrement propices à affaiblir la portée du message paulinien de l'obéissance "par motif de conscience." Outre sa théorie séduisante des quatre royaumes, ce livre raconte le refus de quatre jeunes Hébreux d'adorer la statue d'or érigée par Nabuchodonosor. ${ }^{20} \mathrm{Ils}$ furent jetés dans une fournaise de feu ardent et en sortirent indemnes. Sous Darius le Mède, Daniel fut jeté dans la fosse aux lions pour avoir transgressé un édit de pratique religieuse. ${ }^{21}$ Ces épisodes renforcent la thèse de la résistance à des ordres impies. Althusius y verra même une leçon de gouvernement pour les pays à confessions religieuses multiples. Le code deutéronomique contient de nombreuses prescriptions, morales, religieuses, sociales et rituelles, pour le peuple juif. Une section est consacrée aux devoirs du roi, dans laquelle Yahvé interdit à ce dernier de multiplier le nombre de ses femmes, son or et son argent. ${ }^{22}$

Le texte le plus propre à soulever des controverses est celui du prophète Samuel sur l'établissement de la monarchie. A une époque où l'autorité monarchique occupe tous les esprits, où la contestation vise le détenteur, et parfois la forme, de ce pouvoir, l'épisode narré soulève de graves difficultés pour les consciences chrétiennes favorables à ce type de régime.

Insatisfaits des fils de Samuel établis comme juges, les Anciens d'Israël réclament un roi, à l'instar des autres nations. Cette requête déplait à Yahvé, qui se sent rejeté, mais il commande à Samuel de satisfaire à leur demande, tout en les avertissant de ce que sera "le droit du roi qui va régner sur eux":

\section{Les inconvénients de la royauté.}

Samuel répéta toutes les paroles de Yahvé au peuple qui lui demandait un roi. Il dit: "Voici le droit du roi qui va régner sur vous. Il prendra vos fils et les affectera à sa charrerle et à ses chevaux et ils courront devant son char. Il les emploiera comme chefs de mille et comme chefs de cinquante; il leur fera labourer son labour, moissonner sa moisson, fabriquer ses armes de guerre et les harnais de ses chars. Il prendra vos filles comme parfumeuses, cuisinières et boulangères. Il prendra vos champs, vos vignes et vos oliveraies les meilleures et les donnera à ses officiers. Sur vos cultures et vos vignes, il prélèvera la dime et la donnera à ses eunuques et à ses officiers. Les meilleurs de vos serviteurs, de vos servantes et de vos boeufs, et vos ânes, il les prendra et les fera travailler pour lui. Il prélèvera la dîme sur vos troupeaux et vous-mêmes deviendrez ses esclaves. Ce jour-là, vous pousserez des cris à cause du roi 


\section{4 / Renaissance and Reformation}

que vous vous serez choisi, mais Yahvé ne vous répondra pas, ce jour-là!"

Le peuple refusa d'écouter Samuel et dit: "Non! Nous aurons un roi et nous serons, nous aussi, comme toutes les nations: notre roi nous jugera, il sortira à notre tête et combattra nos combats." Samuel entendit toutes les paroles du peuple et les redit à l'oreille de Yahvé. Mais Yahvé lui dit: "Satisfais à leur demande et intronise-leur un roi." Alors Samuel dit aux hommes d'Israël: "Retournez chacun dans votre ville."”23

Le livre d'Osée apporte un écho à ces propos, car la colère de Dieu devant la demande des Juifs est évoquée. ${ }^{24}$ Les malheurs prédits par Yahvé arrivèrent dès le règne de Saül mais aussi sous d'autres rois de Samarie et de Judée, comme Achab, qui s'empara de la vigne de Naboth, Jézabel, Athalie, Jéroboam Ier ${ }^{25}$

Le passage de Samuel soulève déjà des difficultés dans son contexte. Comme il est précédé par la victoire de Samuel sur les Philistins, il semble que les motifs invoqués par les Juifs soient nuls. La version apparaît alors anti-monarchiste. Mais un passage ultérieur fait état du danger représenté par les Philistins et de la nécessité d'un chef militaire, d'où l'élection de Saül par Dieu pour défendre les Juifs alors affligés et persécutés par leurs ennemis. Cette version, pro-monarchiste, contredit certaines vues de la première. Pour expliquer ces contrastes, des théories de deux ou même de trois sources ont été avancées. A la Renaissance, seule la version défavorable à la monarchie est retenue, sans doute à cause de l'impossibilité d'ignorer cette vive désapprobation. La volonté exprimée par les Juifs est regardée par Dieu comme une véritable apostasie. L'affront subi par Samuel se transforme en offense à Dieu lui-même. Son courroux révèle une préférence pour un modèle théocratique de société. La monarchie constitue une punition infligée aux hommes pour avoir rejeté ce système. Aussi imploreront-ils en vain un retour à la situation antérieure. Mais la surdité divine et la vanité des implorations humaines plaident paradoxalement pour l'inutilité de toute rébellion et de toute résistance, une fois cet ordre instauré.

Le texte biblique aborde indirectement d'autres aspects de la royauté. Les inconvénients annoncés constituent-ils une liste de pouvoirs ou d'abus? Tel est l'enjeu central. Les méfaits décrits sont-ils approuvés, ou voulus? L'absence apparente de limites aux exactions royales estompe la frontière séparant la monarchie de la tyrannie. Le texte indique le rôle du peuple dans ce changement. Les anciens d'Israël formulent la demande. Celle-ci manifeste la double finalité du pouvoir: régir, à savoir juger, puis conduire aux combats. La requête subit aussi l'influence d'un contexte, celui de "toutes les autres nations." La suite du livre précisera les modalités de sélection du roi. Saül est à la fois choisi par Dieu et élu par le peuple, si l'on considère les deux sources ensemble.

Malgré l'importance des textes de saint Paul et de saint Pierre, nous 
limiterons cette étude à l'influence du texte de Samuel dans les écrits politiques à la fin de la Renaissance, réservant pour un autre moment les interprétations des passages du Nouveau Testament.

\section{La tradition critique sur le texte de Samuel: Le Moyen Age}

Déjà au Moyen Age, ce passage de la Bible avait retenu l'attention des Pères de l'Eglise. Saint Grégoire le Grand le commente longuement. ${ }^{26} \mathrm{Il}$ estime qu'à cette occasion, les Juifs ont rejeté la domination spirituelle au profit d'une domination séculière. ${ }^{27}$ Il montre que le déplaisir qu'eut Samuel à cette demande lui vint de ce qu'il anticipait le déplaisir de Dieu. ${ }^{28}$ Pour Grégoire, le discours de Samuel tendait à décourager les Israélites; ${ }^{29}$ ils auront donc bien mérité ce sort, par leur entêtement. Mais ce discours ne signifie pas que les rois doivent tous se conduire de cette façon et Grégoire rappelle l'épisode de la vigne de Naboth pour le démontrer. Néanmoins, comme il s' agit d'un régime où triomphe l'esprit charnel contre le spirituel, il n'est pas étonnant que les rois cherchent à satisfaire leurs appétits, ${ }^{30}$ à oublier les vertus, à perdre leur intégrité. Et parce qu'ils ont maintenu leur demande après avoir été prévenus de ses conséquences, les Israélites ont perdu tout recours contre cette dépravation, tout moyen de revenir en arrière. En même temps, ils ont rejeté l'intermédiaire de Dieu car Samuel était un adjuvant humble et soumis, qui rendait bien la justice et qui leur avait donné la victoire, alors que les rois n'auront pas le même bonheur dans leurs guerres. Semblable rejet attend parfois les fidèles pasteurs ou prélats de la sainte Eglise, car cette audace d'aller contre leur volonté et leurs saints conseils se retrouve encore. Ainsi Grégoire, tout en utilisant la version anti-monarchiste de la Bible, notamment par la mention des victoires remportées par Samuel, ne cherche ni à contester la monarchie ni à légitimer le droit des rois. Le passage de Samuel marque plutôt, comme l'indique la fréquence des termes, un moment historique aux conséquences effroyables, celui d'un passage du spirituel au charnel. ${ }^{31}$ Le rejet de Dieu par les hommes répète l'épisode de la Genèse et entraine une autre forme de châtiment. Parce qu'elles n'apparaissent pas liées à des situations politiques contemporaines, les explications conservent une saveur académique. Les quelques allusions retrouvées chez d'autres Pères de l'Eglise manifestent des préoccupations toujours marginales, dans le prolongement des idées de Grégoire. Chez Cyprien, saint Ignace et saint Chrysostome et dans les Constitutions des Saints Apôtres, le passage sert à illustrer les difficultés que rencontrent parfois les prêtres et les évêques, à cause de l'ingratitude et de l'hostilité des fidèles. ${ }^{32}$ Si l'insulte est parfois leur gain, ils doivent se consoler de ces afflictions à la pensée que Dieu s'irrite et se venge lorsque ses prêtres sont flétris. Comme chez Grégoire, Samuel est traité à l'égal d'un prêtre et la supériorité des prêtres sur les gouvernants s'en trouve confirmée. 
Saint Augustin reste muet sur ce passage dans sa Cité de Dieu. Mais saint Thomas s'en sert pour montrer que la royauté, théoriquement à considérer comme le meilleur régime, peut aisément dégénérer en tyrannie, qui est sa déformation. Saint Thomas signale donc que "dès l'institution, le Seigneur a investi le roi d'un pouvoir tyrannique."33

Les Juifs étaient particulièrement cruels et enclins à la rapacité, et c'est par ces vices surtout que les hommes versent dans la tyrannie. C'est pourquoi le Seigneur ne leur assigna pas dès le début un roi revêtu de l'autorité souveraine, mais un juge et un recteur qui veillât sur eux. C'est plus tard, à la demande du peuple et sous le coup de la colère, qu'il leur accorda un roi, disant clairement à Samuel, comme on le lit au premier livre des Rois $(8,7)$ : "Ce n'est pas toi qu'ils ont écarté, c'est moi, ne supportant plus que je règne sur eux.", 34

Le texte apparait à saint Thomas comme une simple tentative de dissuasion de la part de Samuel:

En fondant l'institution, Dieu ne donnait pas au roi un tel droit. C'est plutôt l'annonce du droit inique usurpé par des rois dégénérés en tyrans et en spoliateurs de leurs sujets. La suite du texte (I Rois 8,17 ) ne permet pas d'en douter: "Et vous serez leurs esclaves"; c'est le caractère même de la tyrannie, puisque les tyrans traitent leurs sujets en esclaves. En parlant ainsi, Samuel voulait donc dissuader le peuple de réclamer un roi; on lit d'ailleurs un peu plus loin $(8,19)$ : "Mais le peuple refusa d'écouter la voix de Samuel"-Malgré tout, il peut arriver, même à un bon roi exempt de tyrannie, d'emmener les jeunes gens, de désigner des chefs de mille et des chefs de cinquante et d'imposer force contributions à ses sujets, en vue d'assurer le bien commun. ${ }^{35}$

Saint Thomas en déduit la supériorité d'un régime monarchique tempéré d'aristocratie, car la situation en Israël prouve que "les dispositions de la loi n'étaient pas satisfaisantes." 36 Celles-ci font pourtant partie du Deutéronome, dont il reprend les idées essentielles pour conclure que les méchants rois supporté par Israël servaient à châtier les révoltes fréquentes du peuple.

Abravanel, humaniste juif né au Portugal en 1437, livre le commentaire le plus exhaustif de ce morceau. ${ }^{37}$ Il l'utilise pour contredire la position des commentateurs bibliques juifs, lesquels estimaient que la Bible contenait des prescriptions impératives pour établir une monarchie. ${ }^{38}$ Abravanel adopte une position assez proche de saint Thomas et il tire de la Bible une leçon défavorable à la monarchie absolue.

\section{Luther et Calvin}

Le renforcement des structures nationales coïncide avec la montée de la Réforme. Le soutien ou la condamnation de celle-ci par les autorités temporelles obligent Luther et Calvin à amorcer une réflexion sur l'ordre 
civil. Les positions de Luther à l'égard de l'état ont fait déjà l'objet de nombreux commentaires. ${ }^{39}$ Rappelons qu'elles ont varié selon les impératifs stratégiques du moment. Par sa théorie des deux glaives, Luther se désintéresse en principe de l' autorité séculière, car le royaume d'ici-bas est disqualifié par rapport à l'au-delà, et il se contente de prôner une obéissance qui n'engage pas l'âme. Les révoltes des paysans, les débordements radicaux de ses disciples, en particulier des anabaptistes, le besoin de la protection des princes l'amènent à préciser sa doctrine, en cinq textes principaux. Intéressé surtout au Nouveau Testament, Luther maintient au coeur des principes régissant les rapports du chrétien à l'Etat l'enseignement de l'épître de saint Paul aux Romains dont il a fait un commentaire. Toutes les admonestations et tous les appels de saint Paul et de saint Pierre à l'obéissance sont confirmée et renforcés, notamment dans son commentaire de l'épître de saint Paul. ${ }^{40}$ Luther néglige le texte de Samuel alors que Calvin, qui traite longuement des gouvernements civils dans le vingtième chapitre de son Institution le cite au moment où il s'interroge sur le meilleur régime. ${ }^{41}$ Il observe qu'il est "bien vray qu'un Roy, ou autre, à qui appartient la domination aisément décline à estre tyran," ${ }^{\prime 42}$ ce qui lui fait favoriser une direction multiple. Comme ses prédécesseurs, il estime qu'un "mauvais Roy est une ire de Dieu sur la terre (Iob, 34, 30; Isa, 3, 4; Osée, 3, 11; Deut. 28, 29.)" "43 Les exhortations de Jérémie de prier pour Nabuchodonosor et pour la prospérité de Babylone servent à faire valoir l'honneur dû à un roi même "pervers et cruel." 44 Abordant les méfaits prévus par Samuel, Calvin les explique ainsi:

\footnotetext{
Certes les Rois ne pouvoyent faire cela justement, lesquels par la Loy estoyent instruits à garder toute tempérance et sobriété(Deut. 17,16 ss.). Mais Samuel appelloit Puissance sur le peuple, pourtant qu'il luy estoit nécessaire d'y obéir, et n'estoit licite d'y résister. Comme s'il eust dit: La cupidité des Rois s'estendra à faire tous ces outrages, lesquels ce ne sera pas à vous de réprimer, mais seulement vous restera d'entendre à leurs commandemens, et d'y obéir. ${ }^{45}$
}

Le respect gardé par David envers Saül témoigne du caractère inviolable de la majesté. "Nous devons tous à noz supérieurs, tant qu'ils dominent sur nous, une telle affection de révérence que celle que nous voyons en David, mesme quelsqui' ils soyent. ${ }^{46}$ Calvin maintient en somme les mêmes exigences de loyauté que Luther, mais à l'aide de références à l'Ancien Testament. Ces longues exhortations à la patience s'achèvent cependant sur un ton ambigu et menaçant, par l'évocation des cas où le meurtrier du roi est l'instrument du châtiment divin et par l'approbation des résistances organisées par les magistrats inférieurs. 


\section{8 / Renaissance and Reformation}

\section{L'exégèse du texte de Samuel entre 1570 et 1625}

Afin de conserver le fil dans le labyrinthe des variations exégétiques autour du texte de Samuel, il conviendrait d'en présenter les données les plus significatives, en tenant compte des intérêts et des factions de l'époque. La première démarche consistera à reconnaitre les positions extrêmes (et non pas extrémistes), c'est-à-dire celles qui s'opposent point par point les unes aux autres et, à partir de ces données, dresser une ligne médiane. Globalement considéré, le texte de Samuel apparaît comme un désaveu par Dieu de la monarchie. Cette position de contestation aurait pu être adoptée par les monarchomaques; elle ne le fut pas. Mais leurs écrits nous permettent de retrouver le camp qui l'adopta. Dans les Mémoires de l'Estat de France, un auteur anonyme tente de définir une position raisonnable en s'attaquant à deux abus présumés: celui des anabaptistes, s'autorisant de ce texte pour rejeter la monarchie, et celui des absolutistes, liant les droits des rois aux conséquences prévues par Samuel. ${ }^{47} \mathrm{Ce}$ renseignement précieux compense faiblement la perte des nombreux écrits des anabaptistes mais il confirme l'existence, autrement hypothétique ou à inférer logiquement, d'une opposition radicale aux énoncés des absolutistes. Mais l'opposition entre les deux interprétations se situe à des niveaux différents. Dans un cas, la désapprobation est déduite de la considération de tout le texte de Samuel, dans l'autre, de la partie intitulée Inconvénients de la monarchie, où Samuel présente comme des droits les malversations exercées par le roi et termine sur l'impossibilité dans laquelle seront les Israélites de se plaindre ou de revenir en arrière. Dans cette perspective, il appert qu'un roi même tyrannique mérite une obéissance sans conditions. Selon l'argumentation anabaptiste, la plus contestatrice, un roi, de l'avis de Dieu, sera toujours un tyran (roi=tyran; toujours et partout). Bodin attribue à Melanchthon un énoncé de ce principe et lui reproche d'avoir tiré cette conclusion du texte de Samuel: "En quoy Melanchthon s'est mespris, qui a pensé que les droits de la majesté soyent les abus \& tyrannies que Samuel dit au peuple en sa harangue." 48

Bodin semble avoir tiré cette réflexion des commentaires de Melanchthon sur les Politiques d'Aristote. Dans ce passage, il est écrit:

Dans les Histoires des Rois, où le droit des rois est décrit par Samuel, les formes les plus acerbes de commandement sont approuvées, ornées de ce titre: que cela soit le droit des rois. L'esprit saint signifie ainsi que le pouvoir légitime, même s'il est dur, est approuvé de Dieu. ${ }^{49}$

Melanchthon se contente donc d'affirmer que Dieu a approuvé les formes les plus pénibles du pouvoir légitime. Son ouvrage contient d'ailleurs des incitations analogues à celles de Luther et de Calvin pour le respect des pouvoir établis. Il y soutient les distinctions habituelles du tyrannicide 
commis par des magistrats en fonction, ou par des particuliers. L'opinion des anabaptistes ne trouve certes pas sa source chez ce théologien, auteur précisément d'un traité Contra Anabaptistas. ${ }^{50}$ Mais les accusations de Bodin s'imposeront plus que les nuances de Melanchthon et Marquez les reprendra sans vérification: "Cete interpretation, comme dit Bodin, est de Melanchthon, \& partant suspecte." 51

Selon les argumentations de caractère absolutiste, Dieu recommande plutôt, dans ce texte comme dans d'autres, d'obéir même à un tyran. Donc à plus forte raison faut-il se soumettre à un bon roi (roi et/ou tyran). Les conséquences de ces interprétations sont aux antipodes. La désapprobation de Dieu d'une forme toujours tyrannique commande son rejet, selon les anabaptistes. La description des droits des rois implique au contraire une obéissance inconditionnelle, selon la thèse opposée. Cette dernière position pourrait être imputée à des absolutistes comme Bodin. Mais dans sa forme extrême, elle est surtout défendue par Jacques Ier, par W. Barclay et par Adam Blackwood, défenseur de Marie Stuart contre Buchanan. Bodin, tout comme vraisemblablement le parti des Politiques qu'il représente, apporte deux réserves. S'il admet qu'on doive respect même à un tyran, il refuse de considérer la liste de Samuel comme celle des droits et encore moins des habitudes d'un roi et il estime qu'il s'agit d'une liste d'abus d'un tyran. Cette position rejoint celle de la plupart des auteurs catholiques.

Comment se définit la place intermédiaire? Elle est d'abord adoptée par les monarchomaques réformés, conformément à leur situation ambiguë entre le respect et la contestation du pouvoir temporel, selon les circonstances. Confiants en la dignité et en la valeur de l'autorité séculière mais parfois forcés de s'y opposer, les monarchomaques jouent sur les deux tableaux et n' excluent aucune partie du texte. Les anabaptistes les obligent à expliquer la colère de Dieu. Et les inconvénients annoncés ne leur paraissent pas des droits reconnus, mais de simples menaces faites par Samuel pour dissuader les Hébreux. Le texte révèle à la fois la facilité avec laquelle un roi devient un tyran (roi $\rightarrow$ tyran) et l'habitude des rois de cette époque et de cette région, l'Asie, de se comporter en tyran (Roi $\rightarrow$ tyran, alors-là). Les monarchomaques en déduisent la nécessité d'un contrôle, d'une Loi ou d'un système capable de brider les rois, comme les Etats. Les monarchomaques catholiques reprennent, quand ils s'intéressent à ce texte, ce qui est rare, cette ligne médiane d'une opposition à certains monarques plutôt qu'à la monarchie..$^{52}$ Ils défendent, à l'instar des scolastiques, les avantages d'une monarchie tempérée, non par les lois fondamentales, comme le souhaitent les Réformés, mais par celles de Dieu et de l'Eglise. Guillaume Rose, dès son premier chapitre, insiste sur la supériorité de la monarchie et renoue ensuite avec la version de saint Cyprien, à l'effet que le passage de Samuel contient des prédictions de ce que Saül 
allait faire, non des prescriptions de ce que les rois peuvent faire.

Le schéma suivant illustre l'éventail des points de vue:

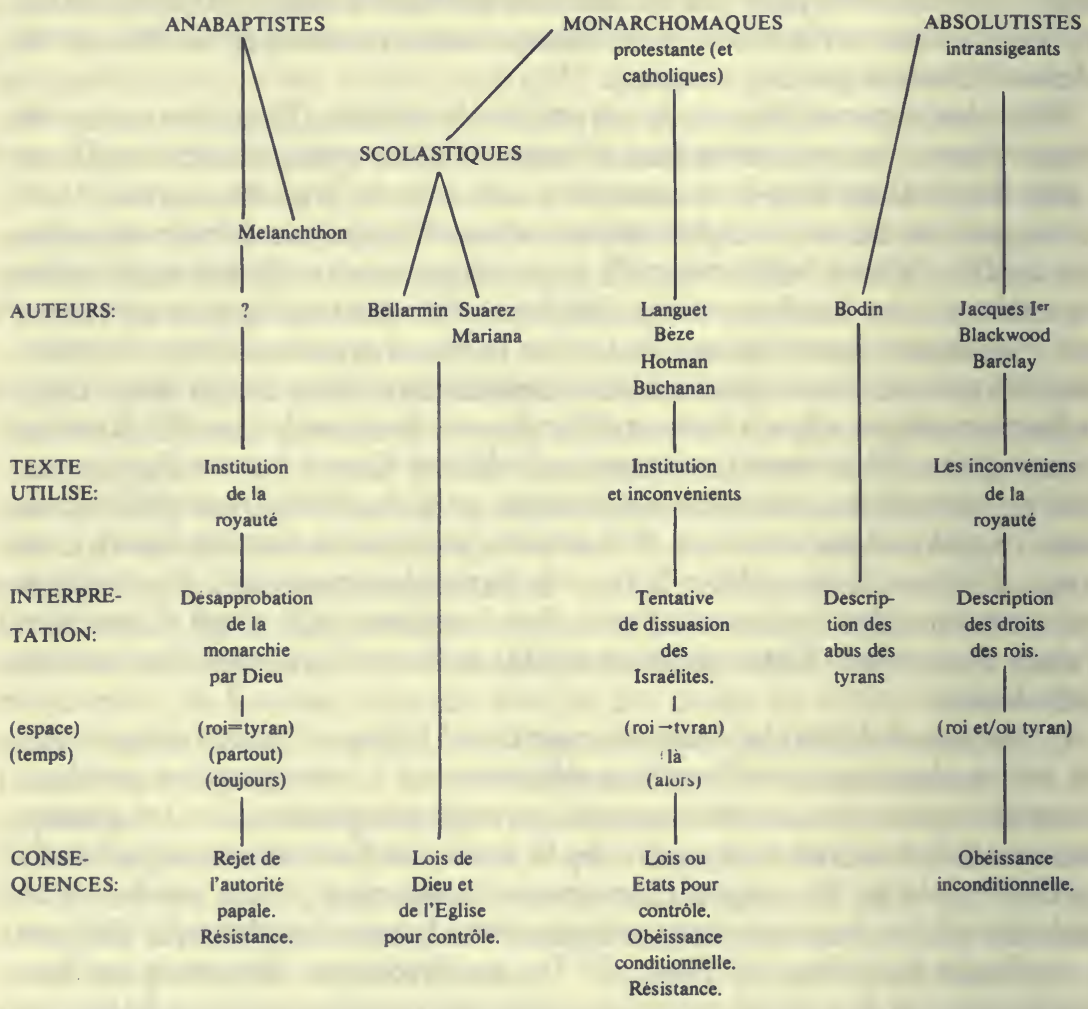

Bien qu'en général fidèles à ces grandes lignes, les interprétations de détail peuvent varier et les catégories sont moins étanches que ne le suggère le schéma. ${ }^{53}$ Rien n'empêcherait par exemple les anabaptistes de relever la propension des rois à devenir tyrans. Les absolutistes de leur côté ont intérêt à reprendre à leur compte l'explication historique souvent présentée chez les monarchomaques et à attribuer la colère de Dieu à des facteurs circonstanciels, ce qui atténue la portée du désaveu. Telle est bien l'argumentation développée par Belloy:

Dont toutefois Dieu ne se courrouça pas contre son peuple; par ce qu'il ne ratiffiast \& n'approuvast l'Estat Royal, mais pour le peu d'asseurance \& deffiance des Israëlites, comme si sa Majesté divine n'eust peu bien disposer de leur Estat, soubs autre police que souz la Royauté: ainsi qu'ils se mescontentoient de Samuel qui s'estoit monstré fidelle serviteur de Dieu \& du public. ${ }^{54}$ 
L'explication est reprise par Jacquer Ier.

La place des scolastiques catholiques reste à déterminer. D'une façon générale, ils pouvaient sans difficultés reprendre l'enseignement des Pères et prendre leurs distances à l'égard de la monarchie. Or la plupart étaient plus favorables à la monarchie, système bien établi, qu'à tout autre régime. A la suite des changements d'allégeance religieuse des souverains, ils durent passer bien contre leur gré dans le camp des ennemis de la royauté, devant la menace d'un roi huguenot en France et sous le règne d'Elisabeth en Angleterre. Leur opinion rejoint alors, même s'ils n'en ont pas toujours conscience, celle des monarchomaques, c'est-à-dire une opposition dirigée bien plus contre la personne du roi que contre la monarchie. Marquez soutient par exemple que les calvinistes utilisent le texte de Samuel pour attaquer la monarchie, ce qui n'est le cas, on l'a vu, que des anabaptistes. La position des scolastiques varie aussi selon la situation géographique des auteurs. L'italien Bellarmin relève simplement qu'il n'a pas plu à Dieu d'accorder un roi à son peuple. Les espagnols Marquez et Ribadeneyra, ardents partisans de la monarchie, adoptent à peu près la solution de Bodin et des monarchomaques, et considèrent le texte comme une série de menaces, non de droits, s'appuyant pour cela sur les arguments de saint Grégoire le Grand. S'opposant à des monarques mais non à la monarchie, ils font valoir les avantages d'un système régi par les lois de Dieu et de l'Eglise. Le jésuite Nicholas Sanders en Angleterre et l'évêque Guillaume Rose en France abondent dans le même sens.

Le livre de Samuel suscite enfin quelques commentaires marginaux. Lipse et Parsons se contentent de relever que la monarchie y est décrite comme la forme de gouvernement la plus répandue, "la plus ordinaire." 55 Surtout les modalités décrites d'accession au pouvoir par Saül servent certaines causes. Enfin, le rôle de Samuel envers le peuple et envers Dieu n'est pas négligé.

\section{Examen détaillé des diverses thèses}

L'argumentation anabaptiste représentée dans le Politique comporte trois motifs pour l'exclusion de la royauté: le refus de Jésus de devenir roi, l'origine violente de la monarchie avec Nemrod, ${ }^{55}$ la colère de Dieu dans Samuel et Osée. Un passage de l'Epittre aux Corinthiens incitant les chrétiens à régler entre eux leurs différends sans d'adresser à des juges couronne cet exposé.

La conviction monarchiste atténuée des monarchomaques est également illustrée dans Le Politique. A l'appui de la thèse reconnaissant la monarchie comme le meilleur des régimes sont citées les opinions des philosophes anciens, les discussions des sages de Perse et de Venise, un extrait de la Genèse sur la domination d'Adam comme modèle d'économie domestique. Les excès sont cependant blâmés, et à propos du droit des 


\section{2 / Renaissance and Reformation}

rois, il est dit que Samuel a été mal interprété "car ce n'est pas une ordonnance, mais une menace, que le peuple aurait au lieu de Roys des superbes tyrans." ${ }^{77}$ Cela s'est vérifié par les règnes d'Achab et de Jézabel. S'intéressant à l'interdiction faite par Jésus à Pierre de tirer son glaive, l'auteur du Politique expose la thèse courante qu'un simple particulier, comme l'était alors l'apôtre Pierre, n'a pas la "vocation légitime"s8 de résister et de prendre les armes.

Bodin s'intéresse peu au texte de Samuel. L'essentiel se trouve déjà exprimé dans sa Méthode. ${ }^{59}$ A son avis, dans les paroles de Samuel "ce n'est pas la royauté, mais bien la tyrannie qui est visée, n'en déplaise à Melanchthon." ${ }^{60}$ Bodin a tout intérét à se démarquer d'un texte si négatif à l'égard des monarques. Dans sa République, il le mentionne surtout pour des problèmes fortuits, comme l'élection ou la succession, les devoirs des rois pour la justice et la guerre; il souligne la popularité du régime attestée par ce passage. ${ }^{61}$ Pour Bodin, Dieu se sent rejeté lorsque ses lieutenants sur terre, rois ou princes, comme l'était Samuel, sont repoussés. ${ }^{62}$ Ses objurgations à l'obéissance enchâssent plutôt des extraits de Jérémie sur Nabuchodonosor. ${ }^{63}$ Il cite le respect de David pour Saül et ajoute:

Il n'y a rien plus frequent en toute l'escripture saincte, que la defense, non pas seulement de tuer, ny attenter à la vie ou à l'honneur du Prince: ains aussi des Magistrats, ores (dit l'Escriture) qu'ils soyent meschans. ${ }^{64}$

La prudence compréhensible de Bodin lui est dictée par son désir de légitimer une monarchie puissante sans en évoquer les risques.

Blackwood, polémisant contre Buchanan, illustre mieux la position des absolutistes. Il s'élève contre la "maligne interprétation" de l'Ecossais et ironisant sur les détours de l'exégèse de cet érudit pourtant grave et expérimenté, il rappelle que les Israélites ont bien demandé un roi, non un tyran, et qu'on ne saurait donc considérer le texte comme une liste d'abus tyranniques mais bien comme des droits royaux légitimes, accordés par Dieu. ${ }^{65}$ Bien sûr, selon Blackwood, cela ne veut pas dire que les rois doivent faire tout cela, mais qu'ils peuvent le faire, quand les temps et les circonstances difficiles le commandent, et il n'y a rien dans cette formulation qui s'oppose aux prescriptions du Deutéronome. Pour Blackwood, le peuple a demandé un roi, Dieu a agréé cette demande et ceux qui régnèrent sur Israël furent des rois, non des tyrans. Ce qui veut dire qu'il faut également supporter des pouvoirs qui ont dégénéré car ils ont reçu l'aval de Dieu. ${ }^{66}$ Blackwood pousse l'interprétation dans un sens si favorable à la monarchie qu'il utilise ce texte pour attribuer au roi la propriété des choses; leur usage et leur possession seuls revenant aux particuliers. ${ }^{67}$ Une telle prétention fait contre elle l'unanimité à cette époque, aussi bien des Politiques que des scolastiques et des monarchomaques, réformés et catholiques. 
Languet, Marquez, Du Perron, l'auteur du “Politique" dans les Mémoires de l'Estat de France et Guillaume Rose sont fort explicites la-dessus. ${ }^{68} \mathrm{Ce}$ dernier par exemple s'indigne qu'une seule phrase de Samuel ait pu laisser croire que les rois pouvaient disposer des biens de leurs sujets, alors que l'indignation du Prophète lorsqu'Achab et Jézabel s'emparent de la vigne de Naboth illustre une situation toute opposée.

P. de Belloy, dans son plaidoyer en faveur d'Henri IV, tombe dans l'ornière absolutiste. L'institution de la royauté chez les Juifs signifie à son avis l'approbation divine de ce régime. Cette institution avait d'ailleurs été annoncée bien avant, lorsque Dieu avait promis à Abraham et à Sara que leurs descendants porteraient le sceptre. William Barclay, autre adversaire déclaré des thèses de Buchanan, suit de très près les énoncés de Blackwood. Il ne voit pas de contradictions dans la Bible, mais une différence de niveau: Samuel montre ce qu'un roi peut faire, le Deutéronome ce qu' un roi doit faire. ${ }^{69}$ Surtout, la réponse du peuple et l'acceptation de Dieu indiquent bien le caractère irréversible de l'institution et l'impossibilité d'échapper aux contraintes parfois dures de la royauté. Barclay consacre plusieurs pages au passage de Samuel, s'élevant notamment contre l'intention de Buchanan de voir dans cette description uniquement des abus, à cette époque de rois généralement bien plus mauvais. Pour Barclay, les rois de ce temps étaient pourtant légitimes et le texte contient le droit royal tel que prescrit au peuple. ${ }^{70} \mathrm{Jacques}$ Ier partage évidemment sans scrupules, en raison de ses fonctions, cet avis et il présente dans le Basilikon Doron une perspective carrément absolutiste. ${ }^{71}$ Ecrivant en 1599 , il a pu connaitre les opinions des monarchomaques et il aborde l'argumentation historique. Comme toute l'Ecriture est inspirée, cela signifie que les paroles de Samuel ne lui sont pas dictées par l'ambition de se maintenir au pouvoir en noircissant les rois, Samuel ne fait pas non plus une prédiction de ce que sera le règne de Saül, puisque ce dernier a été désigné par Dieu pour ses vertus et qu'il n'a commis ensuite aucune action vraiment répréhensible. Il s'agit donc, dit-il, d'un discours pour préparer les peuples à obéir et à ne pas résister même aux tyrans intolérables, car Dieu seul a le pouvoir de faire et défaire les rois. Dieu prévient en effet le peuple qu'il ne servira à rien de murmurer, de rechigner car le renoncement est irréversible. Après ces énoncés généraux, Jacque Ier s'attache au détail des points de justice et d'équité qu'un roi peut transgresser impunément. Si le peuple, après ces avertissements a continué à réclamer un roi, c'est qu'il acceptait ces divers inconvénients pour les avantages retirés au chapitre de la justice et de la guerre. Le texte présente donc l'étendue des pouvoirs possibles du roi, en comparaison desquels ceux des rois chrétiens s' avèrent fort modérés. Donc leur sujets ont encore moins de motifs légitimes pour se rebeller. Jacques Ier complète sa démonstration par les témoignages de respect affichés par David, par les appels de Jérémie, et par une longue 
exposition de l'Epitre aux Romains.

Le Politique résume assez bien les différentes thèses en présence et propose la version soutenue habituellement par les monarchomaques . Ceux-ci, tels Goodman, Buchanan, Bèze et Languet, accordent pour la plupart une attention aussi grande que Jacques Ier au passage de Samuel. Goodman, compagnon d'exil de John Knox à Genève, le cite au complet. Il estime qu'il présente des menaces de punition pour avoir rejeté le règne direct de Dieu:

Wherfore to avoyde the daungers upon both partes, it is more than necessarie that bothe be subjecte to that Rule, and with all diligent care, labour to reteyne it, wherby both maye learne their duetie, and be constrayned justly to execute the same. ${ }^{72}$

Bèze développe la thèse historique à peine esquissée chez Goodman. Au commencement, "l'Eternel seul lui-mesme ... a esté le Monarque. ${ }^{73}$ Malgré leur chance d'avoir un roi "qui ne pouvoit jamais devenir Tyran,"74 les Israélites demandent un roi, alors que l'histoire enseigne qu'il n'y a point de roi qui n'ait abusé de son état:

Le Seigneur, justement irrité contre son Peuple et lui voulant enseigner ce qui lui devoit advenir de ce fol appetit qui les menoit, leur predit par Samuel ce qui est nommé en ceste histoire là, le droit du Roi couché en termes merveilleusement estranges, et portans en somme, que le Roi feroit tout ce qui lui plairoit tant des personnes que des biens de ses subjets: chose vraiment tyrannique et non point Roialle. ${ }^{75}$

Or, selon Bèze, Dieu seul peut exiger quelque chose de sa volonté sans avoir à se justifier, mais la volonté des hommes et des rois doit plier à la raison et être guidée par de bonnes et saintes histoires.

Ceux-là donc se trompent grandement, qui prennent ces parolles de Samuel comme si elles authorisoient les Rois en tout ce que bon leur semble, suivant l'execrable parolle de ceste villaine incestueuse, Si libet, licet, qui n'a esté que trop souvent prattiquee de nostre temps. Ains il faut entendre les parolles de Samuel, comme s'il disoit à Israël, "Vous ne vous contentés point que Dieu soit vostre Monarque, comme il a esté jusques à present d'une façon speciale et particuliere, et voulez en avoir un à la façon des autres peuples. Vous en aurez un donc, mais voici la belle justice qu'il vous fera, et tout le droit duquel il usera envers vous." 76

Bèze y voit enfin la preuve que les magistrats sont issus du peuple et choisis par lui. Quant aux relations entre Saül et David, il reconnait le déférence de ce dernier mais rappelle qu'il s'était armé légitimement, l'onction du Seigneur l'établissant comme "Officier du Roiaume": 
Ce neant moins il appert que son intention a esté de se garantir, voire mesmes par les armes, à l'occasion que dessus. Car autrement, pourquoi se feust-il accompagné de gents de guerre?"1

Buchanan mène conjointement l'analyse des textes de Paul and de Samuel. Amateur de distinguos, il pose ainsi les cadres de son analyse:

I desire you to consider first, what the people requested of God; next, what their reasons were for a new request; and lastly, what was God's answer? ${ }^{78}$

Leur requête, celle d'avoir un roi, était fondée sur la corruptions des fils de Samuel et sur leur désir d'avoir un juge et un chef de guerre. A cette époque régnaient en Asie, sur des peuple à l'esprit plus soumis, des tyrans non limités par les lois. Une telle situation augmenta la colère de Dieu. Le texte décrit les pratiques tyranniques en usage alors, et non les droits des rois, lesquels sont présentés dans le Deutéronome. Et cette dernière liste contredit l'énumération de Samuel. Buchanan balise de la même façon le texte de Jéremie, ou "the prophet does not command the Jews to obey all tyrants, but only the king of the Assyrians. Therefore if, from a single and particular command, you should be inclined to collect the form of a general law, you cannot be ignorant . . . of what an absurdity you will be guilty."79 Car un conseil spécifique ne saurait fonder une loi générale. Enfin, si les Israélites n'ont jamais renversé leurs rois, c'est que Dieu, les établissant, conservait le privilège de les destituer. En d'autres circonstances, les lois et les coutumes des royaumes doivent être respectées. Cela permet à Buchanan de motiver la déposition de Marie Stuart par la noblesse écossaise.

Languet s'arrête avec encore plus de minutie aux éléments du texte et sa synthèse est la plus achevée. Tous les voies ouvertes par les monarchomaques se retrouvent:

- la propriété des biens: non autorisée par le texte.

- le motif de la requête: corruption des fils et exemple des nations voisines.

- l'argument historique: le gouvernement direct de Dieu, indirect par Samuel. Le rejet de Samuel comme rejet de Dieu et sa colère.

- les rôles respectifs du peuple et de Dieu dans le choix du roi: à Dieu l'élection, au peuple l'établissement. Le scénario se répète pour le choix de David, désigné puis confirmé. Cet aspect longuement développé permet l'amalgame des deux sources bibliques contradictoires.

- les buts poursuivis dans l'institution de la royauté: justice et guerre. 
- le recours au texte du Deutéronome pour distinguer droits et abus;

- l'affirmation d'une propension naturelle à la tyrannie. ${ }^{80}$

Languet reconnaît franchement les difficultés posées par cette description d'un roi tyrannique. Déplorant à son tour les interprétations qui légitiment les injustices, il propose la solution habituelle: le peuple ingrat s'est mérité un avertissement.

En somme c'est comme si Samuel eust dit, vous avez demandé un Roy à l'exemple des autres nations, lesquelles pour la pluspart sont mastinees par des tyrans. Vous desirez un Roy qui vous administre justice: mais plusieurs d'entre eux estiment tout ce qu'ils veulent leur estre loisible. Cependant vous delaissez de gayeté de coeur le Seigneur Dieu, la volonté duquel est l'infaillible reigle de justice. ${ }^{81}$

\section{Les scolastiques}

Quant aux interprètes fidèles de la tradition scolastique, ils auront plutôt tendance, comme le rapporte J.A. Maravall pour la période contemporaine à notre étude, à esquiver le texte de Samuel, en raison de leur attachement à la monarchie. ${ }^{82}$ En Espagne, l'un des plus illustres d'entre eux, Suarez, ne s'y intéresse pas. Quant à Mariana, il ne s'y attarde guère, et se contente de dire qu'on aurait tort de s'appuyer sur ce texte pour préférer le régime démocratique au monarchique. Mariana pousse l'esquive à un point rarement atteint, soutenant qu'il en est des formes de gouvernements comme des goûts en matière de vêtements, de chaussures et d'habitation: les meilleures choses plaisent aux uns et déplaisent aux autres. Il conclut que les meilleurs raisons l'inclinent à favoriser le gouvernement d'un seul. ${ }^{83}$ Quelques années auparavant, Ribadeneyra avait été plus sensible aux difficultés inhérentes du texte, en particulier sur le droit de propriété. En s'appuyant largement sur les commentaires de saint Grégoire et de saint Jean Chrysostome, il conteste vigoureusement cette prétention, notamment, comme il est d'usage, par l'exemple de la vigne de Naboth. Il reprend aussi l'argument que certains rois, ainsi que le furent Sennacherib, Nabuchodonosor, Attila et Tamerlan, sont des fléaux de Dieu et les instruments de sa colère, comme le dit le prophète Osée, contre les péchés du peuple. ${ }^{84}$ De tous, Marquez sera le plus prolixe, sans cependant innover beaucoup. Comme Ribadeneyra, il rejette la théorie du droit de propriété des rois sur les biens de leurs sujets en s'appuyant encore sur saint Grégoire mais également sur Bodin, pour lequel le texte relève les abus et non les droits des rois. Plus loin, il justifie la colère de Dieu selon l'argument habituel d'un gouvernement direct de Dieu. Marquez attribue les interprétations abusives d'abord aux courtisans flatteurs, puis dans son second livre, aux calvinistes comme Melanchthon, les premiers se servant de Samuel pour renforcer les pouvoirs du roi, le second pour 
attaquer la "monarchie ecclésiastique," c'est-à-dire de "calomnier la souveraine puissance du vicaire de Jésus Christ." ${ }^{85}$ Marquez représente ainsi le double tranchant du texte, à la fois utile et néfaste à la monarchie. Il fait enfin une recension érudite de tous les penseurs favorables à la monarchie. ${ }^{86}$

En France, le cardinal Du Perron renoue avec une perspective de l'interprétation grégorienne, celle qui consiste à assimiler Samuel à un prélat, ce qui lui permet d'affirmer les droits du pape à déposer les rois. ${ }^{87}$ Mais le détail du texte retient moins l'attention de Du Perron que la généralité des événements et leur similitude avec d'autres dépositions, faites par les prophètes, de Roboam et d'Achab. Cette question du statut "ecclésiastique" de Samuel sépare les absolutistes des scolastiques. Bodin présente toujours Samuel et les Juges comme des magistrats temporels, remplissant des fonctions qui passeront ensuite aux rois. Barclay parlera du mandat extraordinaire de Samuel, qui n'était pas prêtre, argument que Du Perron se sentira obligé d'examiner. ${ }^{88}$

Enfin d'Italie parvient une troisième voix officielle de l'Eglise, celle du cardinal Bellarmin, la plus détachée des controverses et des querelles qui agitent la période étudiée. Bellarmin lit dans toute la Bible une réserve générale de Dieu à l'égard du pouvoir temporel. "Nous devons premierement remarquer, que dès le commencement, il ne luy plût pas que ses fidelles eussent charge des hommes hors de leurs familles, mais seulement du bestail, à cause peut estre du danger qu'il y a de gouverne les peuples." 89

L'attribution du pouvoir à des Juges, et non à des rois, confirme cette méfiance. Aussi lorsque les Israélites demandèrent un roi, Dieu "leur fit parestre en plusieurs façons, qu'il n' avoit pas agreable la resolution qu'ils avoient prize de se soumettre tous à la volonté d'un seul, ainsi que les autres nations avoient fait." ${ }^{\text {" }}$ Dieu leur fit aussi connaitre "combien étoit difficile à supporter le joug que les Roys avoient accoutumé d'imposer à leurs sujets." 91 Et Bellarmin de conclure: "Tout cecy montre évidemment que Dieu n'eust pas agreable que son peuple eust des Roys absolus, ainsi que les infidelles en avoient," car "Il prévoyait qu'ils abuseroient de leur puissance."92 Bellarmin estime que les avertissements de Dieu n'étaient pas vains, comme le prouve le petit nombre de rois élevés à la sainteté (environ 20) depuis l'établissement de l'Eglise, en proportion de celui des évêques (environ 900). Bellarmin, proche des anabaptistes sur l'interprétation générale, ne tire cependant aucune conclusion et n'invite pas au rejet des régimes en place. Par sa simplicité et sa proximité de la Bible, son témoignage apparait paradoxalement comme le plus radical, si bien qu'il aurait été qualifié d'hérétique s'il avait été rendu par une autre personne. 


\title{
La fortune du texte
}

Bien que courte, l'interprétation de Grotius marque un tournant qui achève le cycle des transformations subies par le texte de Samuel. Maravall signale pour la période postérieure à notre étude que les penseurs espagnols du $17 \mathrm{e}$ siècle ont prolongé l'interprétation réservée et plutôt défensive des scolastiques, afin de sauvegarder leur adhésion au régime monarchique. Grotius, quant à lui, inaugure une présentation davantage légaliste:

\begin{abstract}
A l'égard des paroles de Samuel, touchant le droit du Roi, si l'on examine bient le passage, on trouvera, qu'il ne faut l'entendre ni d'un véritable droit, c'est-à-dire, du pouvoir de faire quelque chose honnêtement \& légitimement (car dans l'endroit de la Loi, qui traite des Devoirs du Roi, on lui prescrit une toute autre manière de vivre); ni d'un simple pouvoir de fait (car il n'y auroit-là rien de singulier, puisque les Particuliers se font aussi très-souvent du tort les uns aux autres): mais qu'il s'agit d'un acte, qui, quoi qu'injuste, a quelque effet de droit, je veux dire, qui emporte l'obligation de ne pas résister. C'est pour cela que le Prophète ajoûte, que, quand le Peuple feroit opprimé par les mauvais traitemens du Roi, il imploreroit le secours de Dieu; comme n'ayant alors aucune ressource humaine. C'est donc un droit, dans le même sens qu'il est dit que le Préteur rend justice, lors même qu'il prononce un Arrêt injuste. $^{93}$
\end{abstract}

Il se situe entre ceux qui ne voient dans ce texte qu'une annonce de malheurs et ceux qui prétendent qu'il s'agit d'une liste des droits. Cette voie moyenne est nouvelle, car si elle atténue ou rejette les prétentions juridiques à une puissance illimitée, elle n'en couvre pas moins ses effets et élimine les velléités de résistance à des contraintes fondées sur une légitimité précaire. L'euphémisme "effet de droit" n'empêche donc pas le retour à la vision de Jacques Ier. Au siècle suivant, Pufendorf situera sa position dans la foulée de Grotius et en accentura les conséquences. Samuel avait eu raison, dit-il, de prévenir les Hébreux car un roi, pour son train royal, peut et doit prendre les garçons pour en faire des soldats, les filles pour en faire des cuisinières, parfumeuses, boulangères; il peut aussi s'emparer des champs, des vignes, et des oliviers s'il est pressé par des besoins d'argent. Le discours de Samuel est encore une fois réduit à une paraphrase:

En un mot, si vous voulez avoir un Roi, il faudra que vous l'entreteniez d'une manière convenable à sa dignité \& que vois lui assigniez pour cela certains revenus. Mais, si dans la suite vous venez à trouver ces charges trop pesantes, vous aurez beau souhaitter d'en être délivrez, vous ne pourrez point le détrôner, parce qu'en le choisissant pour vôtre Souverain, vous lui aurez donné un droit, dont il ne vous sera plus permis de le dépouiller sans son consentement. ${ }^{94}$ 
Les seules bornes de ces droits sont la loi ou le droit naturel, ou les conventions établies par les sociétés.

La distribution des résultats de l'interprétation selon un axe délimitant des extrêmes et un centre, illustre l'éventail des exégèses possible entre 1570 et 1625 , et des médiations opérées. Mais les versions moyennes, selon le tableau, n'offrent pas de garanties plus sûres de vérité et cette questions de pertinence ou de justesse ne mérite pas d'être posée. Seule compte la présence des variantes et des invariants d'interprétation. L'écart obtenu et les compromis effectués nous dévoilent la dépendance de l'exégèse des conditions idéologiques globales et historiques particulières. La véritable dichotomie ne se situe pas dans les oppositions de thèses mais entre la volonté invariablement proclamée partout de respecter l'enseignement biblique et cette autre volonté, moins prononcée, parfois occultée, d'accorder le sens de la Bible à des intérêts politiques précis. Révélatrice d'une mauvaise foi inconsciente, cette dichotomie illustre bien la difficulté qu'il y a à accorder un crédit illimité à un texte dont on néglige souvent le propre contexte historique et social. Elle manifeste aussi les obligations faites aux auteurs d'ajuster leurs perspectives ou de défendre leurs intérêts en fonction des contraintes du milieu et de l'évolution, alors accélérée, des conditions politiques. Cet écartement porte les germes d'un progrès car il oblige le lecteur étonné de toute ces divergences à s'interroger sur la capacité d'un livre, dont on prône unanimement le respect le plus total, à régir en détail l'activité humaine. L'influence directe et immédiate de la Bible, comme arbre de vérité, peut se trouver en apparence affaiblie par tous ces courants interprétatifs, mais cet affaiblissement peut renforcer la position de ceux qui sont plus attentifs à l'esprit qu'à la lettre et qui recherchent moins dans la Bible un code qu'une inspiration.

\section{Université d'Ottawa}

\section{Notes}

1 Paroles de Calvin citées par Samuel Berger, in La Bible au XVIe siècle. Etude sur les origines de la critique biblique (Genève: Slatkine Reprints, 1969), p. 38. (Réimp. de l'édition de Paris, 1879).

2 Jean Bodin, Les Six Livres de la République (Lyon: du Puys, 1580), p. 303, (1 éd: Paris, 1576).

3 Ibid., p. 305.

4 Thédore de Bèze, Du droit des magistrats, Intr. et notes de Robert M. Kingdon (Genève: Droz, 1970), p. 24 et ss, ( $1^{\text {e }}$ éd: Genève, 1574).

5 George Buchanan, De jure regni apud Scotos: A Dialogue concerning the Rights of the crown in Scotland, publié au sein de l'ouvrage du Rév. S. Rutherford, Lex, Rex, or the Law and the Prince (Edinburgh; Ogle \& Oliver \& Boyd, 1843), p. 268, (1e éd: Edimbourg, 1579).

6 Hubert Languet, pseud. Etienne Junius Brutus, Vindiciae contra tyrannos, trad. franç. de 1581, édition de A. Jouanna et alii (Genève: Droz, Coll. Les classiques de la pensée politique, 1979), p. 46. Le titre de la traduction française est De la puissance légitime du Prince sur le peuple et du 
peuple sur le Prince. Longtemps considéré comme l'oeuvre de Duplessis-Mornay, le fameux Vindiciae a éte attribué à Languet dans cette édition. Sur la série des diverses attributions, consulter la bibliographie de Myriam Yardeni, La Conscience nationale en France pendant les guerres de religion (1559-1598) (Paris-Louvain: E. Nauwelaerts, 1971), p. 91 et ss. L'article le plus récent sur cette question est de M.P. Raitière, "Hubert Languet's authorship of the Vindiciae contra tyrannos," in Il Pensiero politico, ${ }^{14}$ (1981), (1'e éd: Bâle, 1579).

7 Cf. The Political Works of James I, Intr. de Charles Howard Mcllwain (New York: Russel \& Russel, 1965).

8 Johanes Althusius, Politica Methodice Digesta. Intr. de C.J. Friedrich (Cambridge: At the University Press, 1932). (1e éd: Groningue, 1603).

9 Trad. D. Virion, Nancy, J. Garnich, 1621 (1e éd: Pampelune, 1615).

10 Francisco Suarez, Selections from three works, Ed. par J. Brown Scott, (Oxford: Clarendon Press, 1944), I, 688 et ss.

11 Jacques Davy, cardinal Du Perron, Harangue faicte de la Part de la Chambre ecclésiastique, En celle du tiers Estat, sur l'Article du Serment (Paris: Antoine Estienne, 1615), p. 65. Discours prononcé le 27 octobre 1614 devant le Tiers Etat.

12 Ibid., p. 23.

13 "La Genèse", 4: 17, in La Sainte Bible, traduite en français sous la direction de l'école biblique de Jérusalem (Paris: Ed. du cerf, 1961). Toutes les références et citations ultérieures renverront à cette édition.

14 Cf. Otto Gierke, Political Theories of the Middle Ages, Trad. F.W. Maitland(Cambridge: At the University Press, 1951), p. 89.

15 La Genese, 10, 9-10.

16 Mémoires de l'Estat de France sous Charles neuviesme (Meidelbourg: H. Wolf, t. I, 1576-1577; t. II, 1578; t. III, 1578-1579).

17 Saint Paul, Epitre aux Romains, 13: 1-5.

18 Première epitre de saint Pierre, 2: 13-17.

19 Livre de Jérémie, 27: 1-11.

20 Livre de Daniel, 3: 1-51.

21 Ibid., 6:1-25.

22 Le Deutéronome, 17: 16-19.

23 Premier livre de Samuel, 8: 1-22.

24 De la royauté.

Je vais te détruire, Israël;

qui te pourra secourir?

Ou donc est-il ton roi, qu'il te sauve?

tes chefs, qu'ils te protègent?

ceux-là dont tu disais:

"Donne-moi un roi et des chefs."

Un roi, je te l'ai donné dans ma colère

et, dans ma fureur, je te l'enlève. Osée, 13, 9-11.

25 L'épisode souvent cité de la vigne de Naboth apparaït au Premier livre des Rois, 21: 1-16.

26 Saint Gregoire le Grand, Primum Regum Expositiones, L. IV, chap. I-II, pp. 217-233, in Patrologiae Cursus Completus, Patres latini, Series prima, t. 79, 1849. Le texte de Samuel permet à saint Grégoire de faire de nombreux exposés sur des sujets connexes, comme la distribution des châtiments et des récompenses selon les mérites des hommes, le rôle du Christ dans la rédemption depuis le péché d'Adam, etc.

27 "His autem, qui vivebant sub spiritali regimine, regem petere quid aliud est quam eamdem spiritalem praelationem in saecularem dominationem transferre gestire."Op. cit., p. 219.

28 Grégoire souligne qu'il n'est pas écrit "displicuit sermo Samueli," mais bien "in oculis Samuelis." Ibid. 
29 "Pro reproba voluntate male petentes populi, petitus rex conceditur pro vindicta." Op. cit., p. 221.

30 ". . quia dum reprobam vitam laudant, carnalem mentem tyranne ad exercendam pravitatem roborant." Op. cit., p. 227.

31 Le terme carnalis et ses dérivés sont très souvent utilisés par saint Grégoire.

32 Cf. The Anti-Nicene Fathers (New York: C. Scribner's Sons, 1925). Saint Ignace (I, 60), saint Cyprien (V, 340, 366, 373), Constitutions des Saints Apôtres (VII, 412). Et The Nicene and Post-Nicene Fathers, First Series, saint Chrysostome (XIII, 481).

33 Saint Thomas d'Aquin, Somme théologique. La loi ancienne, t. II, Ia IIae, Q.105, art. 5. (ParisTournai-Rome: Desclée et cie, 1971), p. 238.

34 Ibid., p. 243. La Bible hébraïque comprenait le livre de Samuel et celui des Rois, alors que la version grecque de la Septante intitulait les deux textes Règnes, amalgame repris par la Vulgate latine, citée par saint Thomas, sous le titre de Rois. Les versions modernes retiennent la division du texte hébreu. (Renseignements gracieusement communiqués par le professeur Jean Calloud de Lyon, dont les remarques ont également influencé la présente conclusion.)

35 Ibid., p. 246.

36 Ibid., p. 238.

37 Abravanel, Commentary of the Bible. Texte traduit par Robert Sacks dans Medieval Political Philosophy. A Sourcebook, éd. par R. Lerner et M. Mahdi(Toronto: Collier-Macmillan, 1963), p. 262 et ss.

38 Il écrit: "All of them accepted the notion that there was a positive commandment laid upon Israel to ask for a king. But I am not of this opinion." Op. cit., p. 265.

39 Consulter notamment L.H. Waring, The Political Theories of Martin Luther (New York: Kennikat, 1968); Joël Lefebvre, Luther et l'autorité temporelle (Paris: Aubier Montaigne, 1973); Pierre Mesnard, L'Essor de la philosophie politique au XVIe siecle (Paris: Vrin, 1951), pp. 181 235.

40 Martin Luther, “Commentaire de l'épître aux Romains," 1515-1516, in Luther's Works, éd. par F. Sherman (Philadelphia: Fortress Press), vol 47: "The Christian in Society,” p. 109.

41 Jean Calvin, Institution de la religion chrétienne (Paris: Vrin, 1961), t. IV, Livre IV, chap. XX: "Du gouvernement civil," pp. 510-511. Sur la pensée politique de Calvin, voir aussi MarcEdouard Chenièvre, La Pensée politique de Calvin (Slatkine Reprints, 1970), 383 p.; André Biéler, La Pensée économique et sociale de Calvin (Genève: Librairie de l’Université, 1959), 562 p.; Pierre Mesnard, op. cit., p. 235 et ss.

42 Ibid., p. 512.

43 Ibid., p. 530.

44 Ibid., p. 532.

45 Ibid., p. 531-532.

46 Ibid., p. 533.

47 "Le Politique. Dialogue traittant de la puissance, authorite, \& du Devoir des Princes ..., in Mémoires, t. III, 1578-1579, pp. 69-70.

48 J. Bodin, op. cit., L.I, chap. X, p. 212.

49 "In Historis regum, ubi Samueli jus regni describitur, probatur acerbissima forma imperii, ornatur hoc titulo, quod jus sit regum. Significat enim spiritus sanctus legitimum imperium, quamvis durum sit, tamen Deo probari". Philip Melanchthon, "In Aristotelis aliquot libros, (Philippi Melanchthonis) Commentaria," in Philosophiae Moralis Epitome (Lyon: Apud Seb. Gryphium, 1538), p. 60. La traduction est de nous.

50 Melanchthon s'oppose dans ce texte aux thèses des anabaptistes sur le baptême. Il partage l'opinion de Luther sur l'autorité civile et cite deux fois dans les Loci communes theologici l'epître aux Romains. Il fait cependant suivre le passage de saint Paul d'un extrait des Actes des Apôtres ( 5 , 29), disant qu'il faut obéir à Dieu plutôt qu'aux hommes.

51 J. Marquez, op. cit., p. 156. 
52 Cf. par exemple Guillaume Rose, De Justa Reipub, christianae in reges impios et haereticos authoritate (Anvers: J. Keerbergium, 1592), pp. 8 et 103.

53 Les interprétations des scolastiques et de Bodin seront expliquées plus loin dans le texte.

54 P. Belloy, op. cit., p. 19

55 Juste Lipse, dans ses Conseils et exemples politiques, écrit dans le chapitre "De la principauté" (L. II, I): “Qu'elle est la plus ordinaire. - Pour le temps passé, les histoires sacrees le monstrent, là où les Juifs demandant un Roy, parlent en ceste sorte: Pourvoiez nous d'un Roy, comme TOUTES les nations en sont pourvuës." (Paris: J. Richer, 1606), p. 766. Cette constatation constitue aussi le seul commentaire du texte de Samuel fait par Robert Parsons, jésuite écrivant sur la succession d'Elisabeth, dans $A$ Conference about the next succession to the crowne of Ingland, (s.l.: $\mathbf{R}$. Doleman, 1594). "When the children of Israel did aske a kynge at the hands of Samuel, which was a thousand years before the coming of Christ, they alleaged for one reason that al nations round about them had kings for their governours, and at the very same tyme, the chiefest cyties and commonwealths of Greece," (p. 16).

56 "Le Politique," in Mémoires, t. III, p. 78 et ss. Bodin aussi estime que Nemrod fut le premier qui "assubjectit les hommes par force \& violence." (Op. cit., p. 69). Il soutient deux autres fois dans sa République l'origine violente de la royauté, toujours à l'aide de ce fragment (p. 504 et 511). Pourtant, il est seulement dit dans la Bible que "Kush engendra Nemrod, qui fut le premier potentat sur la terre. c'était un vaillant chasseur devant Yahvé, et c'est pourquoi l'on dit: "Comme Nemrod, vaillant chasseur devant Yahvé." Les prémices de son empire furent Babel, Ereq et Akkad, villes qui sont toutes au pays de Shinéar." Genèse, 10,10. L'interprétation de Bodin est déjà contredite dès cette époque par Marquez, qui rappelle que la Bible qualifie Nemrod de vaillant chasseur et de fondateur de villes, "meu du désir de profiter à tous." Op. cit., p. 23.

57 Op. cit., p. 79. L'auteur du Politique continue ainsi: "Vous tiendrez pour flatteurs (comme dire le vray ils semblent bien en tenir) ceux qui alleguant le droit du Roy recité par Samuel ...." Il est difficile de savoir qui sont ces "flatteurs" favorables aux droits illimités des rois sur les héritages, le bétail, les personnes mêmes, car les écrits de Blackwood et de Jacques Ier sont postérieurs à ce texte.

58 Ibid., p. 125.

59 J. Bodin, La Méthode de l'histoire, Trad Pierre Mesnard (Paris: Les Belles Lettres, 1941). (1éd: 1572).

60 Ibid., p. 275.

61 J. Bodin, La République, pp. 610-611, 970, 988.

62 Ibid., p. 212.

63 Ibid., p. 303-304.

64 Ibid., p. 304-305.

65 A. Blackwood, Adversus Georgii Buchanani dialogum, de jure regni apud Scotos, pro regibus apologia (Pictavis: Apud Franciscum Pagaeum, 1581), pp. 229 et ss.

66 "Nam quae disputantur eo loci, toleranda regum imperia significant, quamvis in tyrannos degenerant, \& benignitate numinis, a quo potestatem acceperunt abutantur." Ibid., p. 238.

67 "Regum enim onia sunt dominio, singulorum usu. Regum sunt omnia proprietate, singulorum possessione." Ibid., p. 232.

68 Cf. surtout H. Languet, op. cit., pp. 162, 174-176; les Mémoires, p. 78; G. Rose, op. cit., p. 101.

69 "Moyses quid debeat Rex facere, Samuel quid posset enunciat." W. Barclay, op. cit., p. 64.

70 “Utrumque jus regni est, \& iisdem Regibus, populoque prescriptum.” Ibid. p. 63.

71 Jacques Ier, "Basilikon Doron," 1599, in op. cit., pp. 213 et ss.

72 Christopher Goodman, How Superior Powers Oght to be obeyed of their subjects, and Wherin they may lawfully by God's Worde be disobeyed and resisted (New York: Columbia University Press, 1931), p. 151. (1e éd: Genève, 1558).

73 T. de Bèze, $D u$ droit, p. 28.

74 Ibid., p. 29. 
75 Ibid., pp. 29-30.

76 Ibid., p. 9.

77 Ibid., p. 22.

78 G. Buchanan, op. cit., p. 268.

79 Ibid., p. 270.

80 H. Languet, op. cit., pp. 180-183.

81 Ibid., p. 182.

82 José-Antonio Maravall, La Philosophie politique espagnole au XVIIe siècle dans ses rapports avec l'esprit de la Contre-Réforme. Traduit et présenté par Louis Gazes et Pierre Mesnard(Paris: Vrin, 1955), pp. 135 et ss.

83 P. Juan de Mariana, Del Rey y de la institucion real, in Bibliotheca de autores españoles (Madrid: Ed. Altas, 1950), t. 31, p. 471.

84 Pedro de Ribadeneyra, Traité de la Religion que doit suivre le prince chrestien et des vertus qu'il doit avoir pourbien gouverner et conserver son Estat. Contre la doctrine de Nicolas Machiavel \& des Politiques de nostre temps, trad. Antoine de Balinghem(Douay: Jean Bogart, 1610), pp. 480 et ss. (1e éd: Madrid, 1595).

85 J. Marquez, op. cit., pp. 141-142.

86 "Philon juif, Platon, Aristote, Seneque, Plutarque, Isocrate, Herodote, Xenophon, Saint Justin martyr, S. Atanaze, S. Cyprian, S. Hierosme, S. Thomas, Bartole, Dion Crisostome \& autres immumerables." Ibid., p. 141.

87 Du Perron, op. cit., pp. 64-65.

88 W. Barclay, op. cit., p. 381. Un autre absolutiste, Belloy, pour montrer que Dieu s'irrite lorsque les rois sont méprisés, cite l'exemple de sa colère lorsque Samuël fut rejeté. Op. cit., p. 45 b.

89 Robert Bellarmin, Le Monarque parfait ou le Devoir d'un prince chrétien, trad. Jean de Lannel (Paris: Sebastien Cramoisy, 1625), pp. 299-300.

90 Ibid., p. 301.

91 Ibid., p. 302.

92 Ibid., p. 305.

93 Hugo de Groot, dit Grotius, Le Droit de la guerre et de la paix, trad. J. Barbeyrac (Basle: E. Tourneisen), t. I, p. 174, (1e éd: Paris, 1625).

94 Samuel de Pufendorf, Le Droit de la nature et des gens, trad. J. Barbeyrac, (Amsterdam: Pierre de Coup, 1734), t. II, p. 369.

N.B.: Les recherches pour la rédaction de cet article one été rendues possibles par une subvention du Conseil de recherches en science humaines du Canada. 